\title{
OTIMIZAÇÃO DE MÉTODO PARA DETERMINAÇÃO DE FLAVONÓIS E FLAVONAS EM FRUTAS POR CROMATOGRAFIA LÍQUIDA DE ALTA EFICIÊNCIA UTILIZANDO DELINEAMENTO ESTATÍSTICO E ANÁLISE DE SUPERFÍCIE DE RESPOSTA
}

\author{
Rosemary Hoffmann-Ribani* \\ Departamento de Engenharia Química, Universidade Federal do Paraná, CP 19011, 81531-970 Curitiba - PR, Brasil \\ Delia B. Rodriguez-Amaya \\ Departamento de Ciência de Alimentos, Faculdade de Engenharia de Alimentos, Universidade Estadual de Campinas, CP 6121, \\ 13083-862 Campinas - SP, Brasil
}

Recebido em 8/7/07; aceito em 7/3/08; publicado na web em 26/8/08

\begin{abstract}
OPTIMIZATION OF A METHOD FOR DETERMINATION OF FLAVONOLS AND FLAVONES IN FRUITS BY HPLC USING STATISTICAL DESIGN AND RESPONSE SURFACE ANALYSIS. This work optimized the HPLC conditions for the simultaneous determination of luteolin, apigenin, myricetin, quercetin and kaempferol in aglycone form, as well defined the best conditions for hydrolysis/extraction of these flavonoids in fruits, using the statistical central composite design and response surface analysis. A reverse phase method was developed using a gradient of methanol/water acidified with $0.3 \%$ formic acid as mobile phase and a photodiode array detector. The samples were extracted with methanol/water $(50: 50 \mathrm{v} / \mathrm{v})$ at $90{ }^{\circ} \mathrm{C}$. The optimum time and $\mathrm{HCl}$ concentration varied for the different fruits investigated, demonstrating the necessity of optimizing these conditions for each fruit analyzed. Good recovery ( 87.1 to $96.3 \%$ ), repeatability and linearity were obtained.
\end{abstract}

Keywords: flavonoids; HPLC analysis; statistical central composite design.

\section{INTRODUÇÃO}

Um aumento na ingestão de frutas e vegetais tem sido correlacionado com a redução do risco de doenças crônicas, como as doenças cardiovasculares e câncer. ${ }^{1} \mathrm{~A}$ atividade antioxidante dos flavonóides, encontrados na maioria das frutas e vegetais, é considerada um dos fatores que contribuem para esta redução. ${ }^{2}$

Os flavonóides constituem uma família de moléculas diversas que consistem de dois anéis aromáticos, que estão conectados por um anel pirano. Estes compostos podem ser agrupados em seis subgrupos principais: flavonas (p.e. apigenina, luteolina), flavonóis (p.e. quercetina, miricetina), catequinas ou flavanóis (p.e. epicatequina, galocatequina), flavanonas (p.e. naringenina, hesperitina), antocianinas (p.e. cianina, pelargonina) e isoflavonas (p.e. genisteina, daidzeina). Das principais classes, os flavonóis glicosídicos predominam em frutas e verduras. ${ }^{3}$

A determinação quantitativa de flavonóides glicosídicos individuais é difícil porque não estão disponíveis para aquisição comercial padrões para a maioria destes compostos. Além disso, mais de 50 glicosídios diferentes para cada um dos flavonóides mais comuns já foram descritos. A hidrólise dos glicosídios para liberar as agliconas oferece um método mais prático para determinação dos flavonóides. ${ }^{4}$

Dependendo do tipo de açúcar ligado ao flavonóide, muitos parâmetros podem influenciar na eficiência da hidrólise como a composição de solventes, tempo de extração, concentração do ácido empregado, temperatura de extração entre outros, podendo ocorrer degradação ou mesmo hidrólise incompleta do glicosídio. Hertog et al. ${ }^{4}$ definiram em seu estudo o uso do ácido clorídrico para hidrólise dos flavonóides, bem como maior eficiência da extração dos flavonóides com solução aquosa a 50\% de metanol. Para otimizar as condições de hidrólise e extração dos flavonóides o emprego de procedimento estatístico se faz necessário, pois leva em consideração as interações destes fatores. ${ }^{5}$

\footnotetext{
*e-mail: ribani@ufpr.br
}

A análise por superfície de resposta (ASR), originalmente descrita por Box e Wilson, ${ }^{6}$ permite a avaliação dos efeitos de dois ou três fatores e suas interações sobre as variáveis de resposta. Assim, ASR é uma coletânea de técnicas matemáticas e estatísticas que têm sido utilizadas com sucesso para desenvolver, melhorar e otimizar processos. ${ }^{7}$ Utilizada com frequiência na modelagem e otimização de processos bioquímicos e biotecnológicos relacionados a sistemas de alimentos, a ASR também tem sido empregada na otimização de metodologias para extração e análise de componentes de alimentos. ${ }^{8}$

No Brasil há uma grande variedade de frutas típicas, tropicais, subtropicais e de clima temperado. Sendo potenciais fontes de flavonóis e flavonas, estudos dos conteúdos nestas se fazem necessários. Somente maçã e laranja foram analisados neste sentido, utilizando um método sem hidrólise. ${ }^{9}$

O objetivo deste trabalho foi definir as condições cromatográficas para a determinação dos flavonóides luteolina (L), apigenina (A), miricetina $(\mathrm{M})$, quercetina $(\mathrm{Q})$ e kaempferol $(\mathrm{K})$ em frutas na forma aglicona, bem como definir as melhores condições da hidrólise/ extração destes flavonóides utilizando ASR.

\section{PARTE EXPERIMENTAL}

\section{Amostras}

Frutas maduras e frescas foram adquiridas em supermercados de Campinas/São Paulo-Brasil. As seguintes frutas foram investigadas: acerola (Malpiglia glaba L.), figo (Ficus carica L.), goiaba (Psidium guajava L.), jaboticaba (Myrciaria jaboticaba Berg), morango (Fragaria ananassa Duch.) e pitanga (Eugenia uniflora L.).

\section{Reagentes}

A água utilizada para o preparo das amostras e fases móveis foi purificada em sistema Milli-Q (Millipore). O metanol e acetonitrila 
de grau cromatográfico e os ácidos acético, fórmico, ascórbico e clorídrico de grau analítico foram adquiridos da Merck KGaA (Darmstadt, Germany). As fases móveis foram filtradas em filtros politetrafluoroetileno (PTFE) da Millipore, com poros de $0,45 \mu \mathrm{m}$ de diâmetro.

Os padrões de miricetina, quercetina, kaempferol, luteolina e apigenina foram adquiridos da Sigma Chemicals Co. (St. Louis, EUA). Soluções estoque dos padrões foram preparadas pela dissolução de cada flavonóide em metanol grau cromatográfico, em concentração de aproximadamente $500 \mu \mathrm{g} \mathrm{mL}{ }^{-1}$, conservadas a $-18^{\circ} \mathrm{C}$ e protegidas da luz. As soluções estoque apresentaram estabilidade superior a 2 meses em $-18{ }^{\circ} \mathrm{C}$. As curvas de calibração foram construídas pela injeção em triplicata de soluções padrões de trabalho em cinco concentrações diferentes. Como altura, forma e tempo de retenção dos picos cromatográficos podem ser afetados pela composição da solução de injeção, preparou-se a solução padrão de trabalho simulando as condições após hidrólise das frutas. Alíquotas da solução estoque de cada padrão foram diluídas em 1,5 mL de água purificada em Milli-Q com $0,08 \%$ de ácido ascórbico. A esta solução foi adicionado $1 \mathrm{~mL}$ de $\mathrm{HCl} 6 \mathrm{~mol} \mathrm{~L}^{-1}$ e o volume foi completado a $5 \mathrm{~mL}$ com metanol (solução padrão de trabalho).

\section{Preparo das amostras}

Ao contrário dos trabalhos publicados, neste estudo foi decidido que as amostras seriam utilizadas na forma natural, sem prévia liofilização. ${ }^{10}$ Durante o processo de liofilização pode ocorrer perda de flavonóides. Além disso, para o cálculo dos teores dos flavonóides nas frutas, a partir dos resultados de amostras liofilizadas, é necessária a determinação do teor de umidade destas. Apesar de ser uma determinação simples, uma imprecisão nas análises de umidade, que está em torno de percentagem $\left(0,01 \mathrm{~g} \mathrm{~g}^{-1}\right)$, pode influenciar significativamente nos conteúdos de flavonóides, que estão em torno de $\mathrm{mg} \mathrm{kg}^{-1}$.

Separadamente, para cada lote de frutas pequenas (acerola, morango, pitanga e jaboticaba) cerca de $3 \mathrm{~kg}$ foram misturados e, por quarteamento, o tamanho da amostra foi reduzido para aproximadamente 800 g. Para cada lote de frutas maiores (goiaba e figo), cada fruta do lote de $3 \mathrm{~kg}$ foi cortada em quatro longitudinalmente e duas porções opostas das frutas, separadamente para cada tipo, foram juntadas. Após a retirada da parte não comestível das frutas, estas foram cortadas manualmente em pequenos pedaços $(1-2 \mathrm{~cm})$. Cada amostra foi inicialmente homogeneizada em multiprocessador de alimentos com $0,135 \%$ (p/p) de ácido ascórbico como antioxidante. Foi então homogeneizada durante 3 min na velocidade $25.000 \mathrm{rpm}$ em homogeneizador do sistema Polytron MR2100, Kinematica-AG (Luzern, Suiça).

\section{Estabelecimento das condições cromatográficas para CLAE}

As análises por CLAE foram conduzidas em cromatógrafo a líquido Waters (Milford, EUA), controlado pelo Software Millennium 32, injetor manual com alça de volume fixo Rheodyne (modelo 7725i), detector de arranjo de diodos modelo 996, a detecção fixada em 370 $\mathrm{nm}$ e espectros na faixa de 200 a $600 \mathrm{~nm}$.

Foi inicialmente utilizada uma coluna Nova Pak C18 (3,9 x 150 $\mathrm{mm}, 4 \mu \mathrm{m})$ e depois definido o uso da coluna Symmetry C18 (2,1 x $150 \mathrm{~mm}, 3,5 \mu \mathrm{m})$, ambas da Waters.

Para definir as condições cromatográficas de separação dos flavonóides agliconas, foi empregada a seguinte estratégia: utilizar uma solução contendo os padrões miricetina, quercetina, kaempferol, apigenina e luteolina, para inicialmente testar, quanto a melhor separação, vários gradientes de fases móveis aquosas com solventes orgânicos, metanol ou acetonitrila, acidificadas com 0,3\% de ácido acético ou ácido fórmico. Posteriormente, extratos hidrolisados das amostras de frutas, com ou sem adição de padrões, foram injetados no cromatógrafo sob as melhores condições conseguidas para os padrões, fazendo os ajustes necessários para a melhor resolução dos picos nas amostras.

\section{Extração e hidrólise dos flavonóides}

Para obter a máxima eficiência de extração e hidrólise sem promover degradação, a hidrólise dos flavonóides glicosídicos à forma aglicona para cada amostra de fruta foi otimizada, utilizandos delineamento estatístico de Composição Central com pontos axiais. ${ }^{7}$ Foram tomadas $15,0 \mathrm{~g}$ de cada fruta homogeneizada com os $0,02 \mathrm{~g}$ de ácido ascórbico já adicionado no preparo da amostra. Dez $\mathrm{mL}$ de $\mathrm{HCl}$ em diferentes concentrações molares iniciais (três níveis codificados em $-1,0$ e 1) e $25 \mathrm{~mL}$ de metanol foram adicionados. A solução de extração assim obtida consistiu de diferentes concentrações molares finais de $\mathrm{HCl}$ em solução aquosa a $50 \%$ em metanol (v/v), com $0,04 \%$ de antioxidante. Após o refluxo a $90{ }^{\circ} \mathrm{C}$ durante diferentes períodos (três tempos codificados em $-1,0$ e 1 ), os extratos foram resfriados e o volume completado a $50 \mathrm{~mL}$ com metanol. Então cada amostra foi filtrada primeiro em peneira de 130 mesh e depois por filtro (PTFE) Millipore de 0,45 $\mu \mathrm{m}$ de diâmetro, antes da análise por CLAE.

\section{Delineamento estatístico}

Um delineamento estatístico de Composição Central (DCC) com pontos axiais foi escolhido para investigar a linearidade ou não dos efeitos dos fatores, concentração do ácido e tempo de hidrólise, na eficiência da extração e hidrólise das agliconas. Cada fator foi testado em três níveis (Tabela 1).

Um DCC com $\mathrm{k}$-fatores e três níveis requer $2^{\mathrm{k}}+2 \mathrm{k}+\mathrm{C}$ experimentos, onde $2^{\mathrm{k}}$ pontos estão nos cantos do quadrado, representando a amplitude (o domínio) do experimento. Os pontos axiais $2 \mathrm{k}$ estão numa distância codificada de $\pm 1,41( \pm \alpha)$ do centro do delineamento medem a possibilidade da não linearidade nos valores obtidos de agliconas em função dos fatores. Os pontos $\mathrm{C}$ em replicatas no centro do quadrado (ponto central) são uma estimativa do erro experimental. Assim, considerando dois fatores e quatro replicatas do ponto central, o planejamento envolveu 12 experimentos, executados em ordem aleatória, que para as amostras de pitanga estão apresentados na Tabela 2.

As primeiras 4 linhas da Tabela 2 são suficientes para a determinação do modelo linear para hidrólise/extração, da linha 5 até a linha 8 do planejamento estão os pontos axiais e as 4 replicatas do experimento, que correspondem ao ponto central, estão da linha 9 até a linha 12 .

\section{Análise de superfície de resposta}

A hidrólise e extração podem ser otimizadas estatisticamente se o valor das concentrações das agliconas dos flavonóides for conhecido como uma função dos fatores experimentais, gerando um modelo matemático. Um gráfico dessa função produz a superfície de resposta para as agliconas, que é obtida aplicando-se regressões lineares múltiplas para os valores obtidos das concentrações em mg $\mathrm{kg}^{-1}$ em função dos fatores experimentais.

A superfície de resposta pode ser representada pelo modelo matemático como segue:

$\mathrm{Y}=\mathrm{b}_{0}+\mathrm{b}_{1} \mathrm{X}+\mathrm{b}_{2} \mathrm{Z}+\mathrm{b}_{11} \mathrm{X}^{2}+\mathrm{b}_{22} \mathrm{Z}^{2}+\mathrm{b}_{12} \mathrm{XZ}$ 
Tabela 1. Fatores e níveis testados (valores codificados estão entre parênteses) para o Delineamento de Composição Central com pontos axiais

\begin{tabular}{|c|c|c|c|c|c|}
\hline \multirow[b]{2}{*}{ Fruta } & \multicolumn{5}{|c|}{ Fatores $\left(\mathrm{mol} \mathrm{L}^{-1}\right)^{\mathrm{a}} / \mathrm{min}^{\mathrm{b}}$} \\
\hline & $\begin{array}{l}\text { Ponto axial inferior } \\
\qquad(-1,41)\end{array}$ & $\begin{array}{c}\text { Nível } \\
\text { inferior } \\
(-1)\end{array}$ & $\begin{array}{c}\text { Nível intermediário } \\
(0)\end{array}$ & $\begin{array}{l}\text { Nível superior } \\
\qquad(+1)\end{array}$ & $\begin{array}{l}\text { Ponto axial supe- } \\
\text { rior }(+1,41)\end{array}$ \\
\hline Acerola (Eugenia uniflora) & $\begin{array}{c}0,2 \mathrm{~mol} \mathrm{~L}^{-1} / \\
15 \mathrm{~min}\end{array}$ & $\begin{array}{c}0,3 \mathrm{~mol} \mathrm{~L}^{-1} / \\
40 \mathrm{~min}\end{array}$ & $\begin{array}{c}0,4 \mathrm{~mol} \mathrm{~L}^{-1} / \\
90 \mathrm{~min}\end{array}$ & $\begin{array}{c}0,5 \mathrm{~mol} \mathrm{~L}^{-1} / \\
140 \mathrm{~min}\end{array}$ & $\begin{array}{c}0,6 \mathrm{~mol} \mathrm{~L}^{-1} / \\
165 \mathrm{~min}\end{array}$ \\
\hline Figo (Ficus carica L.) & $\begin{array}{c}0,6 \mathrm{~mol} \mathrm{~L}^{-1} / \\
45 \mathrm{~min}\end{array}$ & $\begin{array}{l}0,8 \mathrm{~mol} \mathrm{~L}^{-1} / \\
60 \mathrm{~min}\end{array}$ & $\begin{array}{l}1,2 \mathrm{~mol} \mathrm{~L}^{-1} / \\
120 \mathrm{~min}\end{array}$ & $\begin{array}{l}1,6 \mathrm{~mol} \mathrm{~L}^{-1} / \\
180 \mathrm{~min}\end{array}$ & $\begin{array}{l}1,8 \mathrm{~mol} \mathrm{~L}^{-1} / \\
205 \mathrm{~min}\end{array}$ \\
\hline Goiaba (Psidium guajava L.) & $\begin{array}{c}0,12 \mathrm{~mol} \mathrm{~L}^{-1} / \\
15 \mathrm{~min}\end{array}$ & $\begin{array}{l}0,2 \mathrm{~mol} \mathrm{~L}^{-1} / \\
35 \mathrm{~min}\end{array}$ & $\begin{array}{l}0,4 \mathrm{~mol} \mathrm{~L}^{-1} / \\
90 \mathrm{~min}\end{array}$ & $\begin{array}{l}0,6 \mathrm{~mol} \mathrm{~L}^{-1} / \\
140 \mathrm{~min}\end{array}$ & $\begin{array}{l}0,7 \mathrm{~mol} \mathrm{~L}^{-1} / \\
165 \mathrm{~min}\end{array}$ \\
\hline $\begin{array}{l}\text { Jaboticaba (Myrciaria jaboti- } \\
\text { caba Berg) }\end{array}$ & $\begin{array}{c}0,2 \mathrm{~mol} \mathrm{~L}^{-1} / \\
15 \mathrm{~min}^{\mathrm{c}}\end{array}$ & $\begin{array}{c}0,2 \mathrm{~mol} \mathrm{~L}^{-1} / \\
15 \mathrm{~min}\end{array}$ & $\begin{array}{l}0,6 \mathrm{~mol} \mathrm{~L}^{-1} / \\
45 \mathrm{~min}\end{array}$ & $\begin{array}{l}1,0 \mathrm{~mol} \mathrm{~L}^{-1} / \\
75 \mathrm{~min}\end{array}$ & $\begin{array}{l}1,0 \mathrm{~mol} \mathrm{~L}^{-1} / \\
75 \mathrm{~min}^{\mathrm{c}}\end{array}$ \\
\hline Pitanga (Malpighia glabra L.) & $\begin{array}{l}0,2 \mathrm{~mol} \mathrm{~L}^{-1} / \\
15 \mathrm{~min}\end{array}$ & $\begin{array}{c}0,4 \mathrm{~mol} \mathrm{~L}^{-1} / \\
30 \mathrm{~min}\end{array}$ & $\begin{array}{l}1,0 \mathrm{~mol} \mathrm{~L}^{-1} / \\
60 \mathrm{~min}\end{array}$ & $\begin{array}{l}1,6 \mathrm{~mol} \mathrm{~L}^{-1} / \\
90 \mathrm{~min}\end{array}$ & $\begin{array}{l}1,8 \mathrm{~mol} \mathrm{~L}^{-1} / \\
105 \mathrm{~min}\end{array}$ \\
\hline $\begin{array}{l}\text { Morango (Fragaria ananassa } \\
\text { Duch.) }\end{array}$ & $\begin{array}{l}0,6 \mathrm{~mol} \mathrm{~L}^{-1} / \\
35 \mathrm{~min}\end{array}$ & $\begin{array}{l}0,8 \mathrm{~mol} \mathrm{~L}^{-1} / \\
60 \mathrm{~min}\end{array}$ & $\begin{array}{l}1,2 \mathrm{~mol} \mathrm{~L}^{-1} / \\
120 \mathrm{~min}\end{array}$ & $\begin{array}{l}1,6 \mathrm{~mol} \mathrm{~L}^{-1} / \\
180 \mathrm{~min}\end{array}$ & $\begin{array}{l}1,8 \mathrm{~mol} \mathrm{~L}^{-1} / \\
205 \mathrm{~min}\end{array}$ \\
\hline
\end{tabular}

a Concentração do $\mathrm{HCl} \mathrm{mol} \mathrm{L}^{-1}$, na solução de hidrólise. ${ }^{\mathrm{b}}$ Tempo de hidrólise em min. ${ }^{\mathrm{c}}$ Delineamento onde pontos no centro de cada lado do quadrado substituem os pontos axiais.

Tabela 2. Condições experimentais do delineamento estatístico de Composição Central (DCC) com pontos axiais (fatores com valores codificados)

\begin{tabular}{lccccc}
\hline $\begin{array}{l}\text { Experimento } \\
\text { (seqüência }\end{array}$ & \multicolumn{2}{c}{ Fatores (valores codificados) } & \multicolumn{2}{c}{ Concentração de flavonóis em pitanga $\left(\mathrm{mg} \mathrm{kg}^{-1}\right)$} \\
teórica) & Concentração Molar HCl & Tempo de hidrólise (min) & Miricetina & Quercetina & Kaempferol \\
\hline 1 & -1 & -1 & 43,8 & 55,7 & 2,81 \\
2 & +1 & -1 & 24,1 & 49,2 & 2,58 \\
3 & -1 & +1 & 42,5 & 59,9 & 2,94 \\
4 & +1 & +1 & 21,9 & 38,9 & 2,53 \\
5 & $-1,41$ & 0 & 43,9 & 59,3 & 2,08 \\
6 & $+1,41$ & 0 & 15,2 & 32,7 & 1,69 \\
7 & 0 & $-1,41$ & 45,7 & 59,7 & 3,34 \\
8 & 0 & $+1,41$ & 32,1 & 54,1 & 2,81 \\
9 & 0 & 0 & 42,8 & 62,3 & 3,68 \\
10 & 0 & 0 & 42,5 & 60,7 & 3,71 \\
11 & 0 & 0 & 42,6 & 61,5 & 3,69 \\
12 & 0 & 0 & 42,6 & 61,6 & 3,70 \\
\hline
\end{tabular}

$\mathrm{Na}$ Equação 1, a resposta (Y) ou variável dependente foi a concentração em mg kg-1, obtida para cada aglicona dos flavonóides por peso de amostra de fruta. Os valores b são as estimativas dos coeficientes do polinômio, os valores $\mathrm{X}$ e $\mathrm{Z}$ representam os valores codificados dos fatores (tempo de hidrólise e concentração do ácido, respectivamente). Os termos lineares, $\mathrm{b}_{1} \mathrm{X} \mathrm{e} \mathrm{b}_{2} \mathrm{Z}$, são responsáveis pelos efeitos principais, os termos quadráticos, $b_{11} X^{2}$ e $b_{22} Z^{2}$, responsáveis pelos efeitos da curvatura e pelo produto bifatorial dos termos, e $b_{12} \mathrm{XZ}$, é responsável pelos efeitos das interações. Foi realizado teste estatístico de F para determinar a significância dos diferentes modelos obtidos. A análise estatística completa foi conduzida separadamente para a definição da condição de extração e hidrólise de cada tipo de fruta e cada aglicona presente nesta. Todas as análises e testes foram conduzidos utilizando o pacote estatístico Statistica for Windows, versão 6.0 da Statsoft.

\section{Validação do método}

A linearidade do método desenvolvido foi verificada para flavonóis e flavonas na faixa de $0,2-50 \mu \mathrm{g} \mathrm{mL}^{-1}$. As curvas analíticas foram construídas pela injeção em triplicata de soluções padrões de trabalho em cinco concentrações diferentes, baseadas nas faixas esperadas dos seus teores nas amostras de frutas. Os limites de detecção (LD) e quantificação (LQ) foram calculados como a mínima concentração correspondente a 3,3 x (SD/S) e 10 x (SD/S), respectivamente, sendo SD o desvio padrão do branco e $\mathrm{S}$ a inclinação da curva analítica. ${ }^{11}$ Para comprovação prática dos valores calculados, estes foram injetados nas condições estabelecidas para a análise por CLAE. A exatidão e precisão foram verificadas para um nível de concentração dos padrões. Prepararam-se 7 amostras de $15,0 \mathrm{~g}$ de acerola, conforme descrito em Preparo das amostras. Cada uma das amostras foi fortificada com volumes de $300 \mu \mathrm{L}$ de cada uma das soluções estoques dos padrões de miricetina, quercetina e kaempferol e $500 \mu \mathrm{L}$ para os padrões apigenina e luteolina, adicionadas de $10 \mathrm{~mL}$ de $\mathrm{HCl} 2 \mathrm{~mol} \mathrm{~L}^{-1}$ e $25 \mathrm{~mL}$ de metanol em balão de $50 \mathrm{~mL}$ completado com água. A solução aquosa assim obtida, de $0,4 \mathrm{~mol} \mathrm{~L}^{-1}$ de $\mathrm{HCl}$ a $50 \%$ em metanol (V/V) contendo um nível de concentração em torno de $10 \mu \mathrm{g} \mathrm{g}^{-1}$ de acerola para os flavonóis e $17 \mu \mathrm{g} \mathrm{g}^{-1}$ de acerola para as flavonas, foi submetida a refluxo $\left(90{ }^{\circ} \mathrm{C}\right)$ durante 90 min para 
extração e hidrólise dos flavonóides. Os extratos resfriados foram completados a $50 \mathrm{~mL}$ com metanol, filtrados em peneira de 130 mesh e depois por filtro (PTFE) Millipore de 0,45 $\mu \mathrm{m}$ de diâmetro, antes da análise por CLAE.

Todas as determinações nas amostras foram conduzidas em duplicata.

\section{RESULTADOS E DISCUSSÃO}

\section{Condições cromatográficas ótimas para CLAE}

Para a coluna Nova Pak C18 (3,9 X 150 mm, $4 \mu \mathrm{m})$, com volume

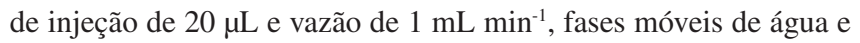
acetonitrila, acidificadas com $0,3 \%$ de ácido fórmico, não separaram os picos de quercetina e luteolina, bem como a apigenina do kaempferol, conforme também relatado por outros autores. ${ }^{4}$ Água e metanol, acidificados com $0,3 \%$ de ácido acético, resultaram em boa separação dos compostos, porém, os picos apresentaram cauda. A melhor separação de padrões foi conseguida com a fase móvel metanol e água acidificados com $0,3 \%$ de ácido fórmico, iniciando na proporção de 20:80, chegando a 45:55 em $5 \mathrm{~min}, 48: 52 \mathrm{em} 17 \mathrm{~min}$ e 20:80 em $20 \mathrm{~min}$, sempre com gradiente linear. Utilizando estas condições para amostras como o morango, ricas em antocianinas, a adição de padrões demonstrou co-eluição de constituintes das amostras com o padrão de miricetina.

A coluna Symmetry C18 (2,1 x $150 \mathrm{~mm}, 3,5 \mu \mathrm{m})$ com volume de injeção de $5 \mu \mathrm{L}$ e vazão de $0,2 \mathrm{~mL} \mathrm{~min}^{-1}$ teve desempenho muito melhor. A melhor separação foi obtida utilizando como fase móvel metanol e água acidificados com $0,3 \%$ de ácido fórmico, iniciando com a proporção de 20:80 passando para 52:48 em 6 min, esta proporção mantida até os 29 min e foi alterada para $72: 28 \mathrm{em} 2 \mathrm{~min}$, permanecendo até aos $40 \mathrm{~min}$. Finalmente, aos $43 \mathrm{~min}$ foi alterada para a composição inicial 20:80, mantida assim até aos $60 \mathrm{~min}$ para re-equilíbrio da coluna antes da próxima injeção. Cromatogramas típicos dos flavonóides agliconas para cada extrato das frutas e para os padrões são apresentados na Figura 1S (material suplementar). Os flavonóides miricetina, quercetina, luteolina, kaempferol e apigenina eluiram nesta sequiência.

\section{Condições ótimas de extração e hidrólise dos flavonóides}

A Tabela 2 apresenta as concentrações em $\mathrm{mg} \mathrm{kg}^{-1}$, obtidas para os flavonóis agliconas em pitanga para diferentes condições experimentais do DCC. Utilizando os valores codificados, foram calculadas por regressões múltiplas as estimativas dos efeitos (parâmetros b) do modelo matemático de regressão (Equação 1) e foram determinados seus erros padrões. Em nível de $95 \%$ de confiança, todos os parâ- metros do modelo para extração/hidrólise foram estatisticamente significativos $(\mathrm{p} \leq 0,05)$ em amostra de pitanga (Tabela 3$)$.

Os cálculos da análise de variância (ANOVA) para os resultados da regressão são apresentados na Tabela 4.

Nos limites do experimento, as três primeiras equações da Tabela 5 representam o modelo experimental da extração/hidrólise de miricetina, quercetina e kaempferol, respectivamente, para amostra de pitanga. As equações estão em função das variáveis estudadas, tempo de hidrólise em minutos (X) e concentração molar do $\mathrm{HCl}$ (Z), sendo $\mathrm{r}^{2}$ o coeficiente de determinação destas.

Aplicando a mesma sequiência analítica por regressões múltiplas aos dados dos DCC, foi possível obter um modelo matemático para cada flavonol aglicona presente nas demais frutas estudadas, considerando os termos lineares, quadráticos e seus produtos. Nos modelos matemáticos refinados obtidos (Tabela 5), em nível de 95\% de confiança, todos os coeficientes foram significativos $(\mathrm{p} \leq 0,05)$. Os valores dos coeficientes de determinação $\left(\mathrm{r}^{2}\right)$ de cada equação gerada variaram de 0,83 a 0,99 . Estes resultados demonstraram que o modelo quadrático seria adequado para descrever as condições de extração/hidrólise. Para verificar o quanto o modelo matemático calculado representa os dados experimentais, foi conduzida análise de variância (ANOVA). As razões entre o quadrado médio da regressão pelo quadrado médio do resíduo $\mathrm{F}_{\mathrm{CAL}}=\left(\mathrm{QM}_{\mathrm{REGRESSÃO}} / \mathrm{QM}_{\mathrm{RESSIDUO}}\right)$, variaram de 15,80 a 80,29 (Tabela 5) para as equações dos modelos de extração/hidrólise dos flavonóis nas demais frutas. Todos valores foram superiores ao valor tabelado de $\mathrm{F}_{5,6}=4,39$ para o nível de $95 \%$ de confiança, indicando equações de regressão significativas, ou a validade do modelo experimental. A boa repetitividade do experimento na análise de cada flavonol pode ser avaliada pelos baixos valores dos erros puros obtidos na análise de variância das regressões para cada fruta, também apresentados na Tabela 5, que variaram de 0,001 a 1,334.

Uma vez que os modelos propostos são válidos, as equações de regressão permitem prever o efeito dos dois parâmetros estudados sobre a extração/hidrólise das agliconas. A relação entre as variáveis independentes e a dependente é representada tridimensionalmente pela superfície de resposta gerada pelos modelos para as agliconas dos flavonóis presentes em cada amostra, conforme a Figura 1, para pitanga e Figura 2S (material suplementar), para as demais frutas.

Constatou-se através da análise de superfície de resposta que as faixas ótimas de trabalho para determinação dos flavonóis em pitanga foram: 0,2 a $1 \mathrm{~mol} \mathrm{~L}^{-1}$ de $\mathrm{HCl}$ e 15 a 80 min de tempo de hidrólise para miricetina , 0,2 a 1,2 $\mathrm{mol} \mathrm{L}^{-1}$ de $\mathrm{HCl}$ e 15 a 105 min de hidrólise para quercetina, e 0,6 a $1,2 \mathrm{~mol} \mathrm{~L}^{-1}$ de $\mathrm{HCl}$ e 15 a 90 min de hidrólise para kaempferol, conforme Tabela 5. Estão apresentadas também na Tabela 5 as faixas para as demais frutas estudadas, obtidas através do mesmo procedimento utilizado para pitanga. Para pitanga que contém os três flavonóis, pela análise da superfície de resposta, as condições

Tabela 3. Estimativas dos coeficientes da regressão do modelo polinomial quadrático, erro puro e significância (p), para a resposta dos teores de flavonóis agliconas em pitanga

\begin{tabular}{|c|c|c|c|c|c|c|c|c|c|}
\hline \multirow[b]{2}{*}{ Coeficiente } & \multicolumn{3}{|c|}{ Miricetina } & \multicolumn{3}{|c|}{ Quercetina } & \multicolumn{3}{|c|}{ Kaempferol } \\
\hline & $\begin{array}{c}\text { Estimativa } \\
\text { Coeficiente } \\
\text { do polinômio }\end{array}$ & Erro Padrão & $\mathrm{p}$ & $\begin{array}{c}\text { Estimativa } \\
\text { Coeficiente } \\
\text { do polinômio }\end{array}$ & Erro Padrão & $\mathrm{p}$ & $\begin{array}{c}\text { Estimativa } \\
\text { Coeficiente } \\
\text { do polinômio }\end{array}$ & Erro Padrão & $\mathrm{p}$ \\
\hline $\mathrm{b}_{0}$ & 42,63 & 0,05 & 4,53E-09 & 61,53 & 0,33 & $3,51 \mathrm{E}-07$ & 3,695 & 0,006 & $8,02 \mathrm{E}-09$ \\
\hline $\mathrm{b}_{1}$ & $-10,12$ & 0,04 & $1,2 \mathrm{E}-07$ & $-8,14$ & 0,24 & $5,37 \mathrm{E}-05$ & $-0,148$ & 0,004 & $4,45 \mathrm{E}-05$ \\
\hline $\mathrm{b}_{2}$ & $-2,83$ & 0,04 & $5,5 \mathrm{E}-06$ & $-1,76$ & 0,24 & 0,004983 & $-0,085$ & 0,004 & $2,29 \mathrm{E}-04$ \\
\hline $\mathrm{b}_{11}$ & $-6,84$ & 0,04 & $5,49 \mathrm{E}-07$ & $-7,93$ & 0,26 & $8,15 \mathrm{E}-05$ & $-0,849$ & 0,005 & $3,3 \mathrm{E}-07$ \\
\hline $\mathrm{b}_{22}$ & $-2,17$ & 0,04 & $1,73 \mathrm{E}-05$ & $-2,44$ & 0,26 & 0,002713 & $-0,251$ & 0,005 & $1,29 \mathrm{E}-05$ \\
\hline $\mathrm{b}_{12}$ & $-0,22$ & 0,05 & 0,026476 & $-3,62$ & 0,33 & 0,001674 & $-0,045$ & 0,006 & 4,17E-03 \\
\hline
\end{tabular}

$\mathrm{p}$ - significância de $5 \%$. 
de máxima extração/hidrólise foram: $0,6 \mathrm{~mol} \mathrm{~L}^{-1}$ por $40 \mathrm{~min} ; 0,6 \mathrm{~mol}$ $\mathrm{L}^{-1}$ por $60 \mathrm{~min}$ e $1 \mathrm{~mol} \mathrm{~L}^{-1}$ por $50 \mathrm{~min}$ para miricetina, quercetina e kaempferol, respectivamente. Optou-se pela condição única de 0,6 mol L ${ }^{-1}$ por $40 \mathrm{~min}$, ideal para extração/hidrólise da miricetina, menos estável dos flavonóis em estudo.

As condições que resultaram na melhor resposta experimental em mg kg-1 , (Tabela 5) para cada fruta foram: acerola, 0,4 mol L-1 por 90 min de hidrólise; figo, $0,5 \mathrm{~mol} \mathrm{~L}^{-1}$ por 160 min de hidrólise; goiaba, $0,6 \mathrm{~mol} \mathrm{~L}^{-1}$ por $55 \mathrm{~min}$ de hidrólise; jaboticaba, $0,6 \mathrm{~mol} \mathrm{~L}^{-1}$ por 45 min de hidrólise; morango, 1,2 $\mathrm{mol} \mathrm{L}^{-1}$ por 120 min de hidrólise; pitanga, $0,6 \mathrm{~mol} \mathrm{~L}^{-1}$ por 40 min de hidrólise.

\section{Desempenho do método desenvolvido}

As curvas analíticas passaram pela origem e apresentaram-se lineares nas faixas de concentração de 0,6 a 23; 0,3 a 48; 0,18 a 37; 1,1 a 26 e 1,6 a $38 \mu \mathrm{g} \mathrm{mL}^{-1}$, respectivamente, para miricetina, quercetina, kaempferol, luteolina e apigenina, apresentando coeficientes de determinação $\left(r^{2}\right)$ respectivos de 0,9995; 0,9999; 0,9997; 0,9989 e 0,9971 .

Os Limites de Detecção (LD), obtidos pelos parâmetros da curva analítica, foram: 0,$86 ; 0,73 ; 0,86 ; 1,03$ e $2,71 \mathrm{mg} \mathrm{kg}^{-1}$, respectivamente, para miricetina, quercetina, kaempferol, luteolina e apigenina.

Tabela 4. Análise de variância (ANOVA) para os flavonóis em amostra de pitanga

\begin{tabular}{|c|c|c|c|c|c|c|}
\hline Flavonol & Fonte de Variação & Soma dos Quadrados & Graus de Liberdade & Quadrado Médio & $\mathrm{F}_{\text {calculado }}$ & $\mathrm{F}_{\text {tabelado }}$ \\
\hline \multirow{5}{*}{ Miricetina } & Regressão & 1182,942 & 5 & 236,588 & 41,57 & $F_{(5,6)}=4,39$ \\
\hline & Resíduo & 34,144 & 6 & 5,691 & & \\
\hline & Falta de ajuste & 34,109 & 3 & & & \\
\hline & Erro puro & 0,035 & 3 & & & \\
\hline & Total & 1217,086 & 11 & & & \\
\hline \multirow{5}{*}{ Quercetina } & Regressão & 1010,076 & 5 & 202,01 & 80,29 & $\mathrm{~F}_{(5,6)}=4,39$ \\
\hline & Resíduo & 15,093 & 6 & 2,516 & & \\
\hline & Falta de ajuste & 13,759 & 3 & & & \\
\hline & Erro puro & 1,334 & 3 & & & \\
\hline & Total & 1025,169 & 11 & & & \\
\hline \multirow{5}{*}{ Kaempferol } & Regressão & 4,865 & 5 & 0,973 & 28,62 & $F_{(5,6)}=4,39$ \\
\hline & Resíduo & 0,206 & 6 & 0,034 & & \\
\hline & Falta de ajuste & 0,205 & 3 & & & \\
\hline & Erro puro & 0,001 & 3 & & & \\
\hline & Total & 5,071 & 11 & & & \\
\hline
\end{tabular}

Tabela 5. Equações de regressão em modelo codificado representando a superfície de resposta dos experimentos em teores dos flavonóis presentes nas frutas estudadas

\begin{tabular}{|c|c|c|c|c|c|c|c|}
\hline FRUTA & Flavonol & $\begin{array}{c}\text { Equação } \mathrm{Y}=(\mathrm{mg} \text { Flavonol kg-1 Fruta) } \\
\text { (Modelo Codificado ) }\end{array}$ & $r^{2}$ & $\mathrm{~F}_{\mathrm{CAL}}$ & $\begin{array}{l}\text { Erro } \\
\text { puro }\end{array}$ & $\begin{array}{l}\text { Faixa ótima } \\
\text { de trabalho } \\
\text { (modelo) }\end{array}$ & $\begin{array}{l}\text { Condições de } \\
\text { hidrólise }\end{array}$ \\
\hline \multirow{3}{*}{$\begin{array}{l}\text { Pitanga } \\
\text { (Eugenia } \\
\text { uniflora L.) }\end{array}$} & M & $\mathbf{Y}=42,63-10,12 \mathbf{X}-2,83 \mathbf{Z}-6,84 \mathbf{X}^{2}-2,17 \mathbf{Z}^{2}-0,22 \mathbf{X Z}$ & 0,97 & 41,57 & 0,035 & $\begin{array}{l}15 \leq \mathbf{X} \leq 80 \\
0,2 \leq \mathbf{Z} \leq 1\end{array}$ & \multirow{3}{*}{$40 \mathbf{X}$ e $0,6 \mathbf{Z}$} \\
\hline & Q & $\mathbf{Y}=61,53-8,14 \mathbf{X}-1,76 \mathbf{Z}-7,93 \mathbf{X}^{2}-2,44 \mathbf{Z}^{2}-3,62 \mathbf{X Z}$ & 0,98 & 80,29 & 1,334 & $\begin{array}{c}15 \leq \mathbf{X} \leq 105 \\
0,20 \leq \mathbf{Z} \leq 1,2\end{array}$ & \\
\hline & $\mathrm{K}$ & $\begin{array}{c}\mathbf{Y}=3,695-0,148 \mathbf{X}-0,085 \mathbf{Z}-0,849 \mathbf{X}^{2}-0,251 \mathbf{Z}^{2} \\
-0,045 \mathbf{X Z}\end{array}$ & 0,96 & 28,62 & 0,001 & $\begin{aligned} 15 & \leq \mathbf{X} \leq 900,6 \\
& \leq \mathbf{Z} \leq 1,2\end{aligned}$ & \\
\hline \multirow{2}{*}{$\begin{array}{l}\text { Acerola } \\
\text { (Malpiglia } \\
\text { glaba L.) }\end{array}$} & Q & $\mathbf{Y}=35,34+0,66 \mathbf{X}-0,36 \mathbf{Z}-3,27 \mathbf{X}^{2}-2,10 \mathbf{Z}^{2}+0,37 \mathbf{X Z}$ & 0,83 & 15,79 & 0,055 & $\begin{array}{r}50 \leq \mathbf{X} \leq 130 \\
0,25 \leq \mathbf{Z} \leq 0,5\end{array}$ & \multirow{2}{*}{$90 \mathbf{X}$ e $0,4 \mathbf{Z}$} \\
\hline & $\mathrm{K}$ & $\mathbf{Y}=15,07-0,92 \mathbf{X}-0,11 \mathbf{Z}-0,10 \mathbf{X}^{2}-0,87 \mathbf{Z}^{2}$ & 0,83 & 18,65 & 0,019 & $\begin{aligned} 15 & \leq \mathbf{X} \leq 90 \\
0,3 & \leq \mathbf{Z} \leq 0,5\end{aligned}$ & \\
\hline $\begin{array}{l}\text { Figo } \\
\text { (Fícus carica } \mathrm{L} .)\end{array}$ & Q & $\mathbf{Y}=14,42-1,86 \mathbf{X}-4,29 \mathbf{Z}-2,20 \mathbf{X}^{2}-2,06 \mathbf{Z}^{2}-3,06 \mathbf{X Z}$ & 0,95 & 23,15 & 0,009 & $\begin{array}{l}45 \leq \mathbf{X} \leq 205 \\
0,60 \leq \mathbf{Z} \leq 1,6\end{array}$ & $160 \mathrm{X}$ e $0,5 \mathbf{Z}$ \\
\hline $\begin{array}{l}\text { Goiba (Psidium } \\
\text { guajava L.)- }\end{array}$ & Q & $\mathbf{Y}=10,74-0,32 \mathbf{X}-0,17 \mathbf{Z}-0,81 \mathbf{X}^{2}-0,28 \mathbf{Z}^{2}-0,60 \mathbf{X Z}$ & 0,97 & 36,27 & 0,031 & $\begin{array}{r}15 \leq \mathbf{X} \leq 110 \\
0,20 \leq \mathbf{Z} \leq 0,7\end{array}$ & $55 \mathbf{X}$ e $0,6 \mathbf{Z}$ \\
\hline $\begin{array}{l}\text { Jaboticaba } \\
\text { (Myrciaria } \\
\text { jaboticaba Berg) }\end{array}$ & Q & $\mathbf{Y}=8,53-0,39 \mathbf{X}-0,51 \mathbf{Z}-0,88 \mathbf{X}^{2}-1,20 \mathbf{Z}^{2}-0,87 \mathbf{X Z}$ & 0,98 & 75,60 & 0,018 & $\begin{array}{c}25 \leq \mathbf{X} \leq 70 \\
0,40 \leq \mathbf{Z} \leq 1,0\end{array}$ & $45 \mathbf{X}$ e $0,6 \mathbf{Z}$ \\
\hline \multirow{2}{*}{$\begin{array}{l}\text { Morango } \\
\text { (Fragaria } \\
\text { ananassa Duch.) }\end{array}$} & Q & $\mathbf{Y}=7,88+0,79 \mathbf{X}-0,40 \mathbf{Z}-1,17 \mathbf{X}^{2}-0,86 \mathbf{Z}^{2}-0,99 \mathbf{X Z}$ & 0,88 & 18,57 & 0,275 & $\begin{array}{r}90 \leq \mathbf{X} \leq 200 \\
0,80 \leq \mathbf{Z} \leq 1,8\end{array}$ & \multirow{2}{*}{$120 \mathbf{X}$ e $1,2 \mathbf{Z}$} \\
\hline & $\mathrm{K}$ & $\mathbf{Y}=11,01-0,43 \mathbf{X}-1,26 \mathbf{Z}-1,42 \mathbf{X}^{2}-1,36 \mathbf{Z}^{2}-1,94 \mathbf{X Z}$ & 0,88 & 19,12 & 0,122 & $\begin{aligned} 100 & \leq \mathbf{X} \leq 205 \\
0,6 & \leq \mathbf{Z} \leq 1,2\end{aligned}$ & \\
\hline
\end{tabular}

$\mathrm{M}=$ miricetina; $\mathrm{Q}=$ quercetina; $\mathrm{K}=$ kaempferol; $\mathrm{X}=$ tempo de hidrólise em min; $\mathrm{Z}=\mathrm{HCl}$ mol L ${ }^{-1}$, concentração na solução de hidrólise; $\mathrm{r}^{2}$ $=$ coeficiente de determinação; $\mathrm{F}_{\text {Calc }}=\left(\mathrm{QM}_{\text {Regressão }} / \mathrm{QM} \mathrm{Resíduo}\right)$. 
A exatidão do método foi demostrada pela média em percentagem dos valores das sete repetições da recuperação dos flavonóides adicionados sobre a amostra de acerola que resultou em 87,1; 94,8; 95,$4 ; 96,3$ e $94,8 \%$, respectivamente, para miricetina, quercetina, kaempferol, luteolina e apigenina para quantidades adicionadas, respectivamente, de 9,$7 ; 10,0 ; 10,2 ; 21,3$ e $14,7 \mu \mathrm{g} \mathrm{mL}^{-1}$. A precisão (repetitividade) foi demonstrada pelos coeficientes de variação das recuperações de 2,$4 ; 2,3 ; 3,1 ; 1,9$ e $1,4 \%$, respectivamente, para miricetina, quercetina, kaempferol, luteolina e apigenina.

\section{Verificação experimental}

Para verificar a performance nas condições definidas pelas análises por superfície de resposta, estas foram aplicadas nas amostras das frutas em estudo, resultando nos valores expressos na Tabela 6. Os teores de quercetina e kaempferol obtidos $\left(\mathrm{Q}=10 \mathrm{mg} \mathrm{kg}^{-1}\right.$ e $\mathrm{K}=9,6$ $\mathrm{mg} \mathrm{kg}^{-1}$ ) para amostra de morango, são comparáveis aos relatados por Franke et al. ${ }^{12}\left(\mathrm{Q}=9 \mathrm{mg} \mathrm{kg}^{-1} \mathrm{e} \mathrm{K}=6 \mathrm{mg} \mathrm{kg}^{-1}\right)$, Häkkinen e Törrönen ${ }^{13}$ $\left(\mathrm{Q}=3\right.$ a $5 \mathrm{mg} \mathrm{kg}^{-1}$ e $\mathrm{K}=2$ a $\left.9 \mathrm{mg} \mathrm{kg}^{-1}\right)$ e Hertog et al..$^{14}(\mathrm{Q}=7,7$ a $10 \mathrm{mg}$ $\mathrm{kg}^{-1}$ e $\mathrm{K}=7$ a $16 \mathrm{mg} \mathrm{kg}^{-1}$ ). Em todas amostras estudadas não foram detectadas as flavonas apigenina e luteolina.
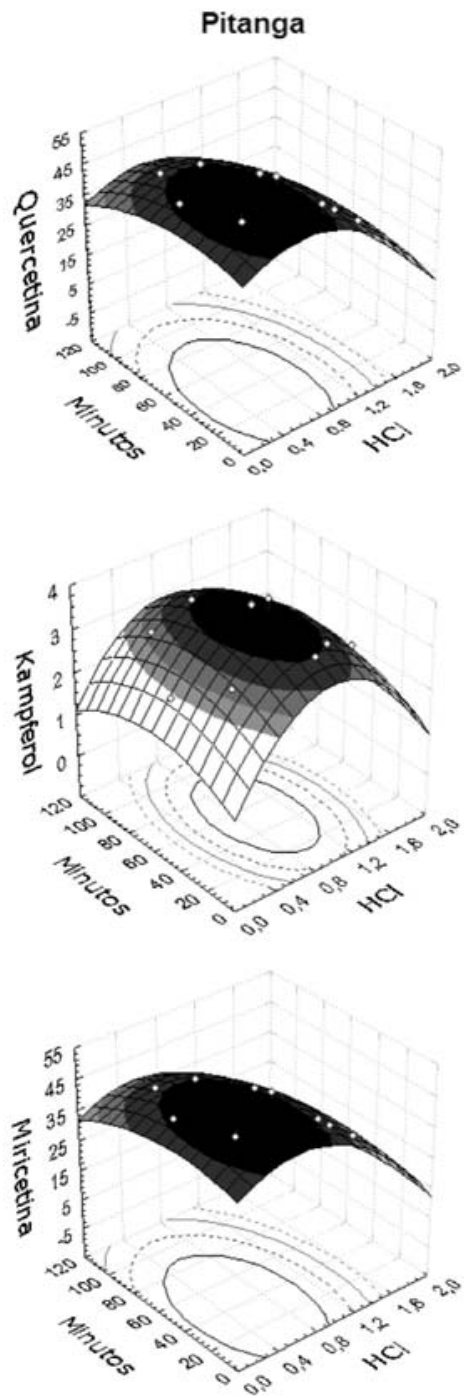

Figura 1. Superfícies de resposta na determinação das agliconas ( $\left.\mathrm{mg} \mathrm{kg}^{-1}\right)$ de quercetina, kaempferol e miricetina, em amostra de pitanga em função da concentração do $\mathrm{HCl}\left(\mathrm{mol} \mathrm{L}^{-1}\right)$ e tempo de hidrólise em minutos
Tabela 6. Conteúdo de flavonóis em frutas analisadas segundo as condições estabelecidas neste estudo

\begin{tabular}{lccc}
\hline \multirow{2}{*}{ Fruta } & \multicolumn{3}{c}{ Concentração $\left(\mathrm{mg} \mathrm{kg}^{-1}\right)^{\mathrm{a}}$} \\
& Miricetina & Quercetina & Kaempferol \\
\hline $\begin{array}{l}\text { Acerola (Eugenia } \\
\text { uniflora } \text { L.) }\end{array}$ & nd & $41 \pm 0$ & $11 \pm 0$ \\
$\begin{array}{l}\text { Figo (Ficus carica } \text { L.) } \\
\text { Roxo }\end{array}$ & nd & $13 \pm 0$ & nd \\
$\begin{array}{l}\text { Goiaba (Psidium } \\
\text { guajava L.) Ogawa } \\
\text { (vermelho) }\end{array}$ & nd & $11 \pm 1$ & nd \\
$\begin{array}{l}\text { Jaboticaba (Myrciaria } \\
\text { jaboticaba } \text { Berg) }\end{array}$ & nd & $8,7 \pm 2,8$ & nd \\
$\begin{array}{l}\text { Pitanga (Malpighia } \\
\text { glabra } \text { L.) }\end{array}$ & $42 \pm 2$ & $67 \pm 2$ & $4,1 \pm 1,0$ \\
$\begin{array}{l}\text { Morango (Fragaria } \\
\text { ananassa } \text { Duch.) Oso } \\
\text { Grande }\end{array}$ & nd & $10 \pm 0$ & $9,6 \pm 0,7$ \\
\hline
\end{tabular}

${ }^{a}$ Média \pm desvio padrão ( $\mathrm{mg} \mathrm{kg}^{-1}$ de fruta natural), análise em duplicata; nd = não detectado; $\mathrm{LQ}=$ Limite de quantificação: miricetina $=2,6 \mathrm{mg} \mathrm{kg}^{-1}$; quercetina $=2,3 \mathrm{mg} \mathrm{kg}^{-1}$; kaempferol $=2,6 \mathrm{mg} \mathrm{kg}^{-1}$. $\mathrm{LD}=$ Limite de Detecção: miricetina $=0,86 \mathrm{mg} \mathrm{kg}^{-1}$; quercetina $=$ $0,73 \mathrm{mg} \mathrm{kg}^{-1}$; kaempferol $=0,86 \mathrm{mg} \mathrm{kg}^{-1}$.

\section{CONCLUSÕES}

As melhores condições cromatográficas para análise dos flavonóides agliconas em frutas foram: coluna de fase reversa Symmetry C-18 (2,1 x $150 \mathrm{~mm}, 3,5 \mu \mathrm{m})$, volume de injeção de $5 \mu \mathrm{L}$; fase móvel de metanol e água acidificados com $0,3 \%$ de ácido fórmico, em gradiente linear e vazão de $0,2 \mathrm{~mL}$ por minuto. A alta correlação dos modelos definidos pela análise de superfície de resposta demonstrou que o modelo polinomial de segunda ordem pode ser usado para otimização das condições de extração e hidrólise dos flavonóides em amostras de frutas. As condições ótimas de extração e hidrólise diferiram entre as frutas, demonstrando a necessidade de otimizar as condições para cada fruta sob investigação.

\section{MATERIAL SUPLEMENTAR}

As figuras citadas no texto: $1 \mathrm{~S}$ (Cromatogramas típicos dos flavonóides agliconas dos extratos das frutas e padrões) e 2S (Superfícies de resposta na determinação das agliconas em morango, acerola, goiaba, figo e jaboticaba), estão disponíveis para consulta gratuita em http:// quimicanova.sbq.org.br, na forma de arquivo PDF.

\section{AGRADECIMENTOS}

Ao PICDT / UFPR pela concessão de bolsa à primeira autora, à FAPESP e ao CNPq pelo apoio financeiro para execução do trabalho através dos projetos PRONEX ${ }^{\circ}$ 2003/10151-4 e Projeto Universal $\mathrm{n}^{\circ} 477189 / 2004-0$, respectivamente.

\section{REFERÊNCIAS}

1. Armstrong, B. K.; Mann, J. L.; Adelstein, A. M.; Eskin, F.; J. Chron. Dis. 1975, 28, 455; Block, G.; Patterson, B.; Subar, A.; Nutr. Cancer 1992, 18, 1; Burr, M. C.; Sweetnam, P. M.; Am. J. Clin. Nutr. 1982, 36, 673; Phillips, R. L.; Lemon, F. R.; Beeson, W. L.; Kuzma, J. W.; Am. J. Clin. Nutr. 1978, 31, 191; Verlangieri, A. J.; Kapeghian, J. C.; El-Dean, S.; Bush, M.; Med. Hypotheses 1985, 16, 7. 
2. Castellucio, C.; Paganga, G.; Melikian, N.; Bolwell, G. P.; Pridham, J.; Sampson, J.; Rice-Evans, C.; FEBS Lett. 1995, 368, 188; Hertog, M. G. L.; Feskens, E. J. M.; Hollman, P. C. H.; Katan, M. B.; Kromhout, D.; Lancet 1993, 342, 1007; Keli, S. O.; Hertog, M. G. L.; Feskin, E. J. M.; Kromhout, D.; Arch. Int. Med. 1996, 156, 632; Knekt, P.; Jarvinen, R.; Reunanen, A.; Maatela, J.; Br. Med. J. 1996, 312, 478; Middleton, E.; Rice-Evans, C.; Miller, N. J.; Bolwell, G. P.; Bramley, P. M.; Pridham, J. B.; Free Radical Res. 1995, 22, 375; Rimm, E. B.; Katan, M. B.; Ascherio, A.; Stampher, M. J.; Willet, W. C.; Ann. Int. Med. 1996, 125, 384.

3. Kühnau, J.; World Rev. Nutr. Diet. 1976, 24, 117; Markham, K. R.; Meth. Plant Biochem. 1989, 1, 197; Williams, C. A.; Harborne, J. B. Em The Flavonoids. Advances in Research since 1986; Harborne, J.B., ed.; Chapman \& Hall: London, 1994.

4. Hertog, M. G. L.; Hollman, P. C. H.; Venema, D. P.; J. Agric. Food Chem. 1992, 40, 1591.

5. Haaland, P. O.; Experimental Design in Biotechnology, Marcel Dekker: New York, 1989.

6. Box, G. E. P.; Wilson, K. B.; J. Roy. Statist. Soc. 1951, B13, 1.
7. Myers, R. H.; Montgomery, D. C.; Response Surface Methodology: Process and Product Optimization using Designed Experiments, $2^{\text {nd }}$ ed., Wiley: New York, 2002; Barros Neto, B.; Scarminio, I. S.; Bruns, R. E.; Planejamento e Otimização de Experimentos, $2^{\mathrm{a}}$ ed., Educamp: Campinas, 1995.

8. Cacace, J. E.; Mazza, G.; J. Food Sci. 2003, 68, 240; Careri, M.; Elviri, L.; Mangia, A.; Musci, M.; J. Chromatogr., A 2000, 881, 449; Catharino, R. R.; Godoy, H. T.; Ciênc.Tecnol. Aliment. 2001, 21, 326; LiyanaPathirana, C.; Shahidi, F.; Food Chem. 2005, 93, 47.

9. Arabbi, P. R.; Genovese, M. I.; Lajolo, F. M.; J. Agric. Food Chem. 2004, 52, 1124.

10. Franke, A. A.; Custer, L. J.; Arakaki, C.; Murphy, S. P.; J. Agric. Food Chem. 2004, 17, 1; Häkkinen, S.; Törrönen, R.; Food Res. Int. 2000, 33, 517; Hertog, M. G. L.; Hollman, P. C. H.; Katan, M. B.; J. Agric. Food Chem. 1992, 40, 2379.

11. Shabir, G. A.; J. Chromatogr., A 2003, 987, 57.

12. Franke, A. A.; Custer, L. J.; Arakaki, C.; Murphy, S. P.; J. Agric. Food Chem. 2004, 17, 1.

13. Häkkinen, S.; Törrönen, R.; Food Res. Int. 2000, 33, 517.

14. Hertog, M. G. L.; Hollman, P. C. H.; Katan, M. B.; J. Agric. Food Chem. 1992, 40, 2379. 


\section{OTIMIZAÇÃO DE MÉTODO PARA DETERMINAÇÃO DE FLAVONÓIS E FLAVONAS EM FRUTAS POR CROMATOGRAFIA LÍQUIDA DE ALTA EFICIÊNCIA UTILIZANDO DELINEAMENTO ESTATÍSTICO E ANÁLISE DE SUPERFÍCIE DE RESPOSTA}

Rosemary Hoffmann-Ribani*

Departamento de Engenharia Química, Universidade Federal do Paraná, CP 19011, 81531-970 Curitiba - PR, Brasil Delia B. Rodriguez-Amaya

Departamento de Ciência de Alimentos, Faculdade de Engenharia de Alimentos, Universidade Estadual de Campinas, CP 6121, 13083-862 Campinas - SP, Brasil
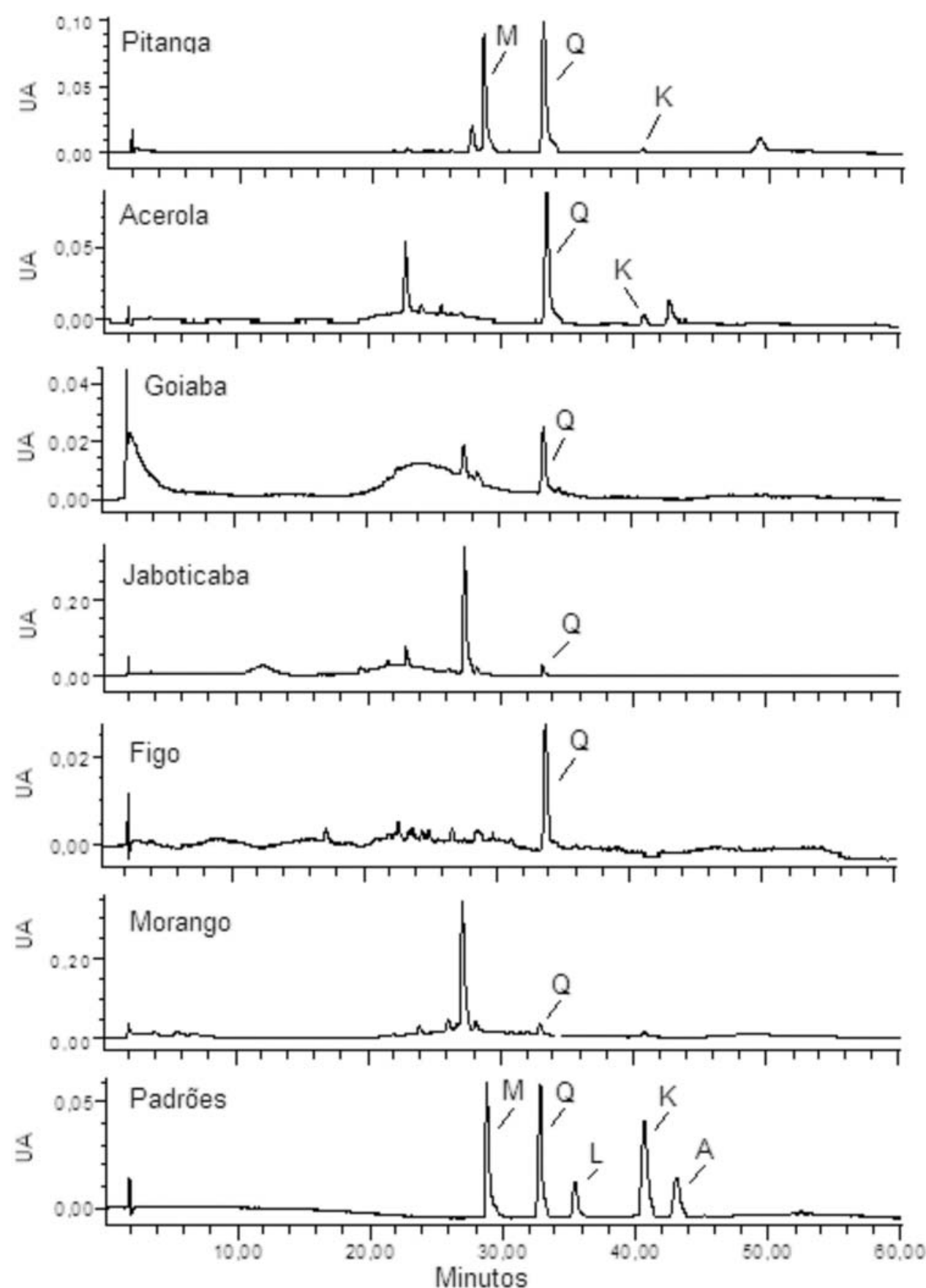

Figura 1S. Cromatogramas típicos dos flavonóides agliconas de extratos hidrolisados de pitanga, acerola, goiaba, jaboticaba, figo, morango e dos padrões, obtido com coluna Symmetry C18 (2,1 x $150 \mathrm{~mm}, 3,5 \mu \mathrm{m}) . M=$ miricetina, $Q=$ quercetina, $K=$ kaempferol, $L=l$ luteolina, $A=$ apigenina. Fase móvel, metanol e água acidificados com 0,3\% de ácido fórmico, iniciando com a proporção de 20:80 passando para 52:48 em 6 min, esta proporção mantida até os 29 min, então alterada para 72:28 em 2 min. Finalmente aos 43 min foi alterada para a composição inicial 20:80, mantida assim até aos 60 min 


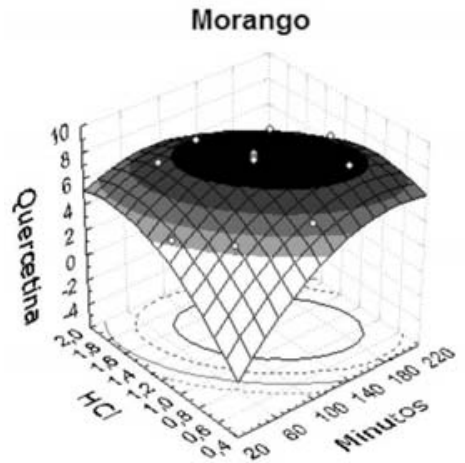

Acerola

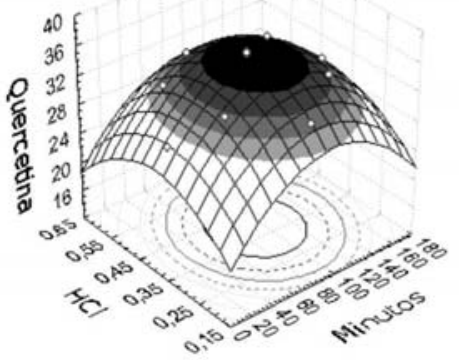

Goiaba

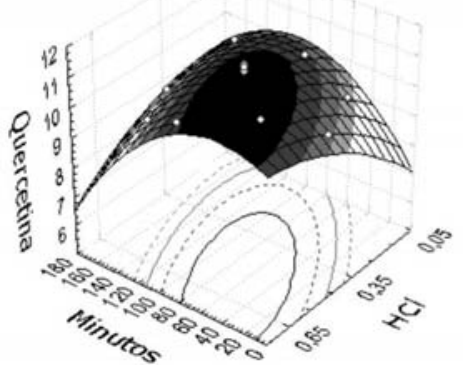

Morango

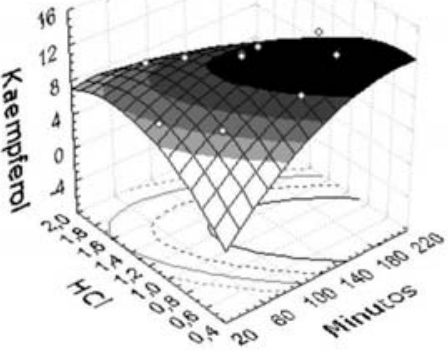

Acerola

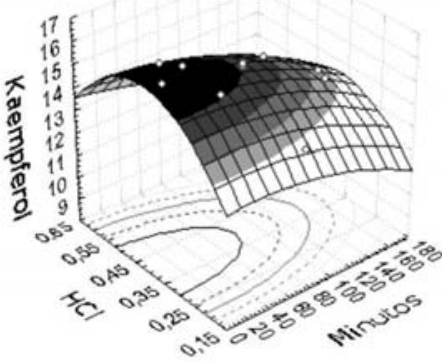

Figo

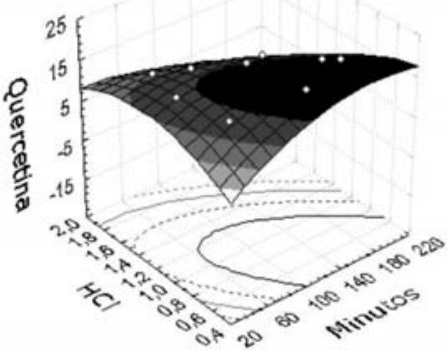

Jaboticaba

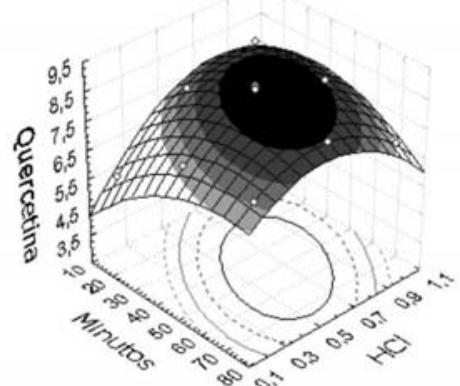

Figura 2S. Superfícies de resposta na determinação das agliconas $\left(\mathrm{mg} \mathrm{kg}^{-1}\right)$ de quercetina e kaempferol em amostras de morango e acerola e de quercetina em goiaba, figo e jaboticaba, em função da concentração do $\mathrm{HCl}\left(\mathrm{mol} \mathrm{L}^{-1}\right)$ e tempo de hidrólise em min 